\title{
Reconstruction of a pathologic fracture following osteomyelitis of the mandible using a fibula osteocutaneous flap
}

Taeki Kim ${ }^{1}$,

Junhyung Kim ${ }^{1}$,

Jaehoon Choi ${ }^{1}$,

Taehee $\mathrm{Jo}^{1}$,

Hyeong Chan Shin',

Woonhyeok Jeong ${ }^{1}$

Departments of ${ }^{1}$ Plastic and

Reconstructive Surgery and ${ }^{2}$ Pathology,

Dongsan Medical Center, Keimyung

University School of Medicine, Daegu, Korea
The use of a fibula osteocutaneous flap is currently the mainstay of segmental mandibular reconstruction. This type of flap is used to treat tumors, trauma, or osteoradionecrosis of the mandible. However, a fibula osteocutaneous flap may also be a good option for reconstructing the mandible to preserve oropharyngeal function and facial appearance in cases of pathological fracture requiring extensive segmental bone resection. Chronic osteomyelitis is one of the various causes of subsequent pathologic mandibular fractures; however, it is rare, and there have been few reports using free flaps in osteomyelitis of the mandible. We share our experience with a 76-year-old patient who presented with a pathologic fracture following osteomyelitis of the mandible that was reconstructed using a fibula osteocutaneous flap after wide segmental resection.

Keywords: Fracture, spontaneous / Free tissue flaps / Mandible / Osteomyelitis

\section{INTRODUCTION}

Osteomyelitis of the maxillofacial area is uncommonly encountered in clinical practice [1]. Most cases of osteomyelitis occur in the mandible, with osteomyelitis of the maxilla accounting for only $1 \%$ to $6 \%$. It mainly occurs due to odontogenic causes such as dental extractions, surgeries, and facial traumas [2]. Advanced mandibular osteomyelitis, which fails to heal despite appropriate treatment, can subsequently cause pathologic fractures, thereby requiring reconstruction after segmental resection. In this case, use of the fibula osteocutaneous flap represents an appropriate reconstruction method to provide func-

Correspondence: Woonhyeok Jeong

Department of Plastic and Reconstructive Surgery, Dongsan Medical Center, Keimyung University School of Medicine, 1035 Dalgubeol-daero, Dalseo-gu, Daegu 42601, Korea

E-mail: psjeong0918@gmail.com

This work was supported by the National Research Foundation of Korea (NRF) grant funded by the Korea government (MSIT) (No. 2021R1G1A100455611).

Received December 22, 2020 / Revised March 25, 2021 / Accepted April 19, 2021 tional and aesthetic results.

Here, we present our experience with a case of reconstruction of a pathologic fracture following osteomyelitis of the mandible using a fibula osteocutaneous flap in a 76-year-old man.

\section{CASE REPORT}

A 76-year-old man with a mandibular pathologic fracture visited our department after referral for mandibular reconstruction from the dental department. Upon presentation, the alveolar bone of the right mandibular body was partially exposed through a mucosal defect, but the lesion was nonsuppurative due to the administration of antibiotics at a local hospital before the patient arrived at our medical center (Fig. 1A). Approximately 2 months earlier, dental extraction of the right lower first molar was performed at a local dental clinic, and the symptoms began at that time. Computed tomography $(\mathrm{CT})$ revealed an irregular bony fracture with sequestrum in the right mandibular body accompanied by mild soft tissue thickening of the 

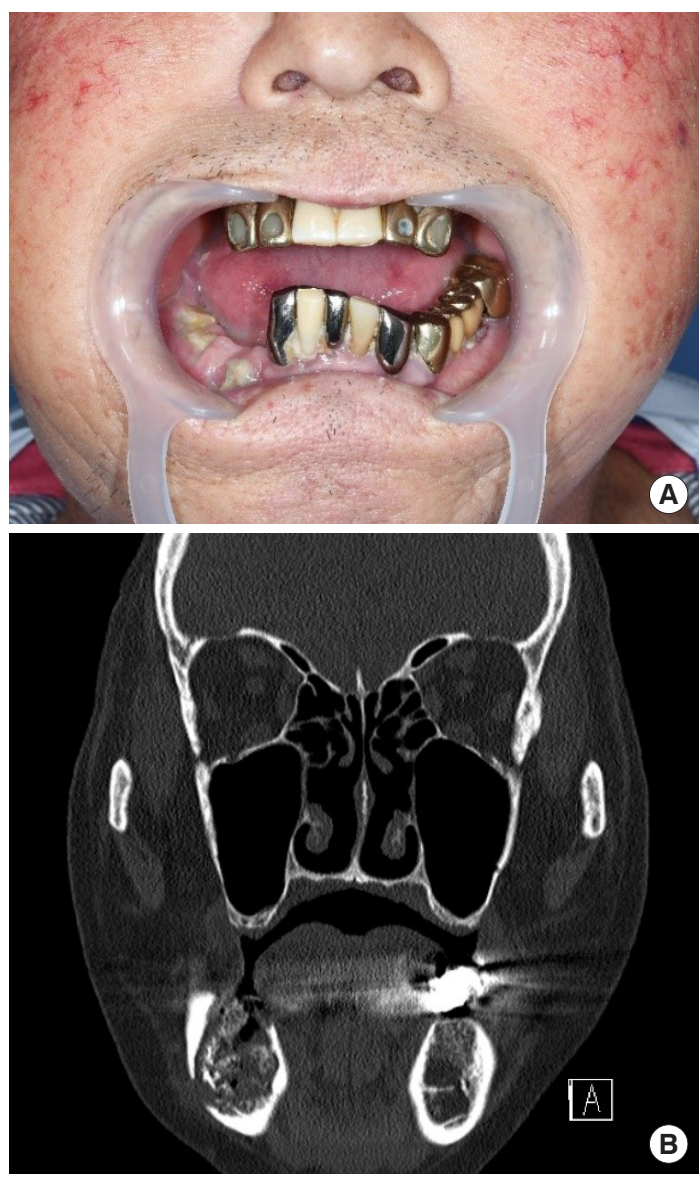

Fig. 1. Preoperative photograph and computed tomography image. (A) The mucosal defect with exposed alveolar bone is observed at the right mandibular body area. (B) Preoperative computed tomography imaging revealing an irregular bony fracture with sequestrum at the right mandibular body.

chin, and several reactive lymph nodes were noted at level I in the right neck (Fig. 1B). Chronic osteomyelitis of the mandible was suspected. However, the range of pathologic fracture was too large to consider osteomyelitis as the primary cause, and based on the daughter's claim of his medical history of osteoporosis, the possibility of bisphosphonate-related osteonecrosis of the jaw (BRONJ) was considered. Surgical resection of the lesion and reconstruction using a fibula osteocutaneous flap were planned to manage the wide bony discontinuity after resection.

To increase the reconstruction accuracy and reduce the operation time, a three-dimensional (3D) printed modeling method was used. Before surgery, a 3D model of the patient's skull was printed based on CT imaging, and the area expected to be resected from the symphysis to the right angle was cut off. The part to be reconstructed with a fibula free flap was composed of resin and fixed on both sides. Using this model as a template, prebending of the reconstruction plate was performed, and its position was checked in advance based on the cutting edge, an-

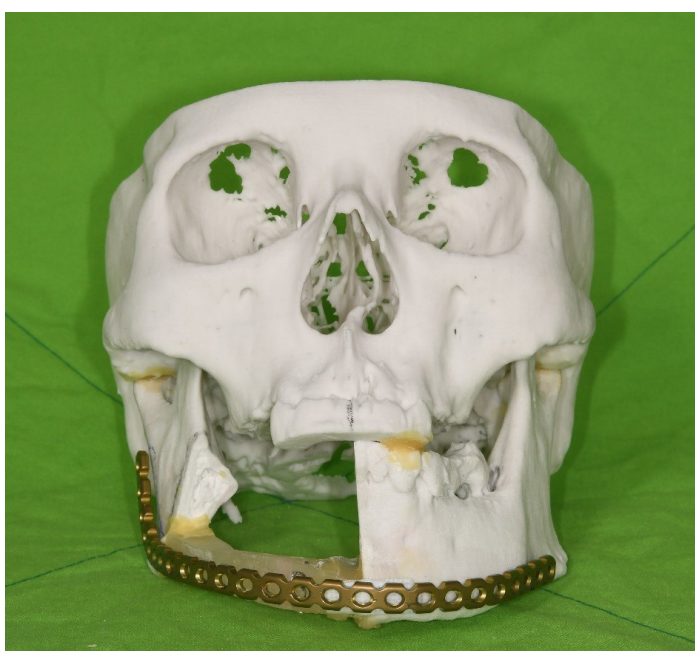

Fig. 2. Prebent reconstruction plate based on a three-dimensional printing model of the patient's skull. The expected resection site was cut off and connected with a resin template.

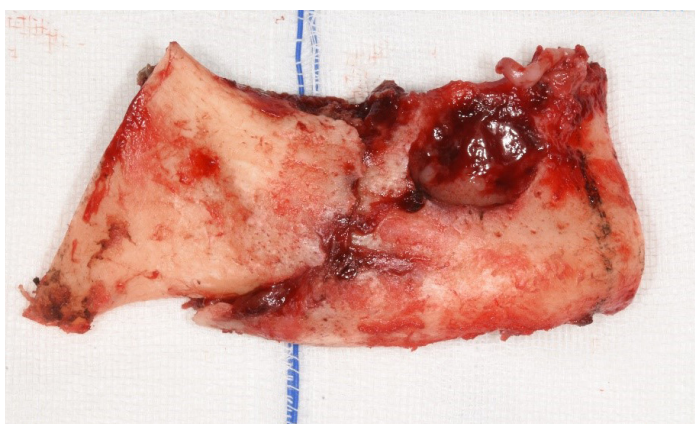

Fig. 3. Segmental resection was performed.

gle, and inferior border of the printed mandible (Fig. 2).

During surgery, the resection margin was set to ensure that enough viable bone was preserved on both sides, and surgical resection of the mandibular segment, including the extensive osteonecrotic lesion and the pathologic fracture, was performed between the symphysis and right angle (Fig. 3). After harvesting the right fibula osteocutaneous flap, osteotomized fibula segments were fixed to the prebent plate. The reconstruction plate was then fixed to both residual mandibular ends with three screws each and additional four-hole miniplates. The ipsilateral facial artery and vein were chosen as the recipient vessels. Microvascular anastomoses were performed with end-toend sutures in arteries and vein couplers for venous anastomosis. The intraoral mucosal defect could be completely covered with the harvested skin paddle. On postoperative day 2, flap congestion was observed, and venous thrombosis could be found upon exploration immediately in the operating room. Venous reanastomosis was performed end-to-side to the external jugular vein. After revision surgery, the viability of the flap 


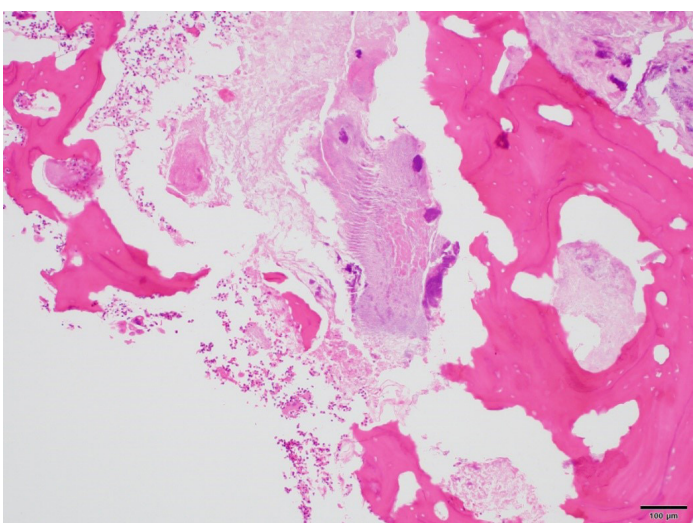

Fig. 4. Histological staining showing bone fragments (with empty osteocyte lacunae), acute inflammatory cells, and bacterial colonies $(\mathrm{H} \& \mathrm{E}, \times 100)$.

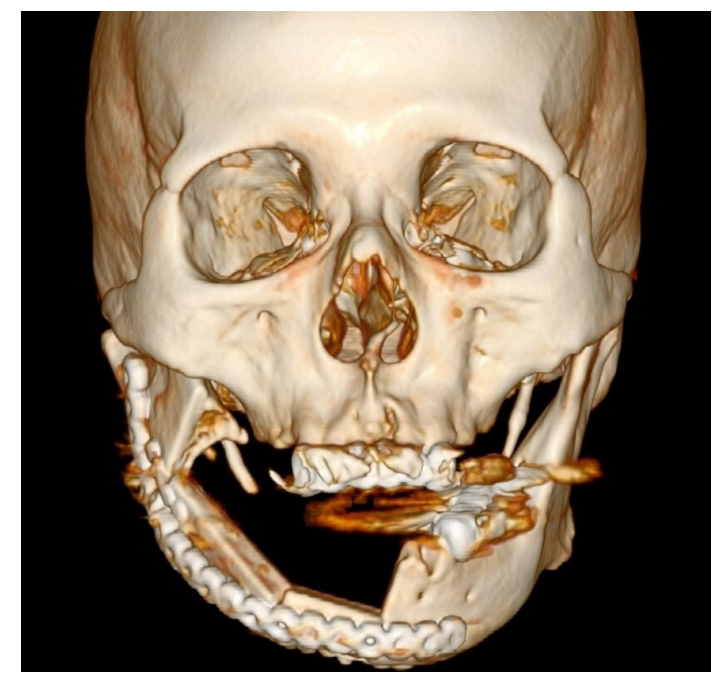

Fig. 5. Three-dimensional computed tomography scan at postoperative day 50 . Bony continuity from the symphysis to the right angle is successfully achieved.

was adequately maintained. The resected segment included sufficient healthy bone around the necrotic lesion; therefore, we did not perform any tissue culture. Instead, we used third-generation cephalosporin as a prophylactic antibiotic therapy.

We checked the patient's local hospital records and medication history, but we could not find any evidence of him taking bisphosphonates. Histological examination of the resected bone segment showed necrotic bone tissue with empty osteocyte lacunae and abscesses with bacterial colonies (Fig. 4). Considering the abovementioned findings along with the preoperative CT results, the final diagnosis was pathological fracture following chronic osteomyelitis of the mandible.

CT performed on postoperative day 50 showed successful osseous continuity (Fig. 5). Although the postoperative course was complicated by his poor condition, at 2 months after sur-
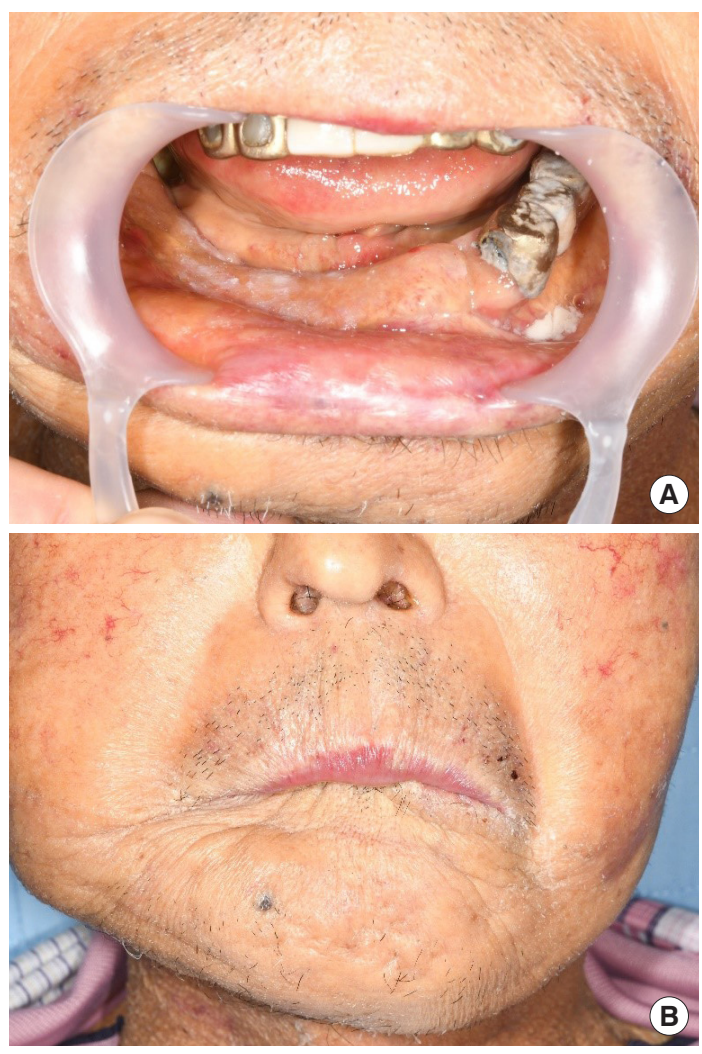

Fig. 6. Two-month postoperative photographs showing (A) the mucosal defect covered with a stabilized skin flap and (B) successful reconstruction.

gery, the patient was discharged with good occlusion and a stabilized skin flap (Fig. 6).

\section{DISCUSSION}

Pathologic fractures are defined as fractures occurring in weakened bone due to an underlying pathological effect rather than by trauma. They rarely occur in the mandibular area, accounting for less than $2 \%$ of all mandibular fractures $[3,4]$. Various factors can cause pathological fracture, including osteomyelitis, osteoradionecrosis, BRONJ, surgical interventions such as tooth extraction, and tumors. These various etiologies make it challenging to treat. In addition, poor nutrition and systemically immunocompromised status can facilitate pathologic fracture [5].

BRONJ was first described by Marx in 2003 [6]. Bisphosphonates are commonly prescribed to manage various conditions, such as osteoporosis and metastatic bone disease, due to their bone-resorption inhibition effect. Despite their well-established beneficial effects, various side effects can also occur, with the most serious being osteonecrosis of the jaw [7]. The incidence of BRONJ after taking oral bisphosphonates is $0.01 \%$ [8], and 
tooth extraction is the most common trigger (48.1\%), followed by apical periodontitis (13.5\%) and marginal periodontitis (10.8\%) [9]. For diagnosis, all of the following should be met: current or previous history of treatment using bisphosphonates, exposed jawbone in the oral cavity for more than 8 weeks, and absence of a history of radiation therapy [8]. In this study, according to his daughter, the patient had a history of osteoporosis; thus, we assumed that he was taking some bisphosphonates. As the symptoms appeared after tooth extraction, we considered BRONJ, but it was excluded after working through the differential diagnosis; however, it is important to verify whether the patient is taking medications such as bisphosphonates or denosumab when osteonecrosis of the jaw is encountered.

Osteomyelitis of the jaw is defined as inflammation and infection of the cancellous and cortical portion of the maxillary skeleton and was first described by Pell et al. in 1955 [1]. In clinical practice, maxillofacial osteomyelitis is rarely reported, and the incidence has been dramatically decreased since the introduction of antibiotics and improvements in oral hygiene [10]. Dental extraction, surgical interventions of the mandible, and maxillofacial trauma can be odontogenic causes, and related comorbidities include malnutrition, alcoholism, smoking, diabetes mellitus, hypertension, osteoporosis, and steroid use [11]. A recent report has shown that osteomyelitis may also be accompanied by necrotizing fasciitis of the masticator space without evidence of an odontogenic origin [12]. The diagnosis can be made based on the patient's history, clinical examination, radiologic findings, and histopathologic examination. In the acute stage, patients can complain of pain, swelling, fever, and paresthesia in the affected area, and fistula formation, lymphadenopathy, and pathologic fracture can be found incidentally. Chronic osteomyelitis of the mandible is defined as osteomyelitis that persists for over 4 weeks. Its pathogenesis is poorly understood, and its clinical and radiologic appearances are variable [2].

In this case, the patient took oral steroids because of adrenal insufficiency and had a frequent intra-articular injection history due to osteoarthritis of the knee. Considering that osteomyelitis can occur when the wound from a tooth extraction is infected due to persistent periodontal or periapical infection that spreads to the underlying bone [2], extraction of his lower first molar 2 months earlier was thought to be the cause. Furthermore, the aforementioned steroid use would have facilitated the occurrence of pathologic fracture from chronic osteomyelitis lesions. Its rare incidence and the paucity of studies dealing with its management make treatment difficult. The basic principle in treating chronic mandibular osteomyelitis is the eradication of pathogenic microorganisms and the removal of all affected tissues. Most cases can be treated with small resections, but in intractable cases, as in our study, wide resection with reconstruction should be performed [10].

There have been few reports on the management of isolated osteomyelitis of the mandible using free tissue flaps. The main goal of mandibular reconstruction is to maintain oropharyngeal function and facial appearance, thereby preventing a dramatic loss in quality of life [13]. The fibula osteocutaneous flap was popularized by Hidalgo in 1989 [14]. Recently, new reconstruction methods have been introduced, such as using prefabricated titanium implants, which are safe and effective in terms of operation time and donor site morbidity [15], but fibular osteocutaneous flaps are still the first option for mandible reconstruction because they can provide extended lengths of not only healthy soft tissue but also bicortical bone and have a long vascular pedicle with low donor site morbidity. Its failure rate is relatively high due to technical difficulties caused by the size discrepancy between the lower extremity and cervical vessels [16]. But with careful selection of recipient vessels the need for revision surgery can be reduced with improved outcomes, especially with superficial thyroid arteries and internal jugular veins [17]. Using virtual planning and a 3D printed modeling with a prebent plate, as in our case, it is possible to improve the accuracy of the reconstruction and reduce the operation time [18]. In addition to its use for tumors or osteoradionecrosis, if a large segmental bone resection is required due to pathologic fracture caused by osteomyelitis of the mandible, the fibula osteocutaneous flap could be a suitable reconstructive method with good aesthetic and functional results.

\section{NOTES}

\section{Conflict of interest}

No potential conflict of interest relevant to this article was reported.

\section{Ethical approval}

The study was approved by the Institutional Review Board of Keimyung University Dongsan Medical Center (IRB No. 202012-036) and performed in accordance with the principles of the Declaration of Helsinki. Written informed consent was obtained.

\section{Patient consent}

The patient provided written informed consent for the publication and use of his images.

\section{ORCID}

Taeki Kim 
Junhyung Kim https://orcid.org/0000-0001-9077-2853

Jaehoon Choi https://orcid.org/0000-0001-6180-1619

Taehee Jo https://orcid.org/0000-0001-8671-6429

Hyeong Chan Shin https://orcid.org/0000-0002-3716-7678

Woonhyeok Jeong

\section{Author contribution}

Conceptualization: JK. Supervision: WJ. Writing - original draft: TK. Writing - review \& editing: JC, TJ, HCS, WJ.

\section{REFERENCES}

1. Pell GJ, Shafer WG, Gregory GT, Ping RS, Spear LB. Garre’s osteomyelitis of the mandible; report of case. J Oral Surg (Chic) 1955;13:248-52.

2. Schuknecht B, Valavanis A. Osteomyelitis of the mandible. Neuroimaging Clin N Am 2003;13:605-18.

3. Coletti D, Ord RA. Treatment rationale for pathological fractures of the mandible: a series of 44 fractures. Int J Oral Maxillofac Surg 2008;37:215-22.

4. Choi BJ, Choi SC, Kwon YD, Drew SJ. Aneurysmal bone cyst causing a pathologic fracture of the mandibular condyle. J Oral Maxillofac Surg 2011;69:2995-3000.

5. Boffano P, Roccia F, Gallesio C, Berrone S. Pathological mandibular fractures: a review of the literature of the last two decades. Dent Traumatol 2013;29:185-96.

6. Marx RE. Pamidronate (Aredia) and zoledronate (Zometa) induced avascular necrosis of the jaws: a growing epidemic. J Oral Maxillofac Surg 2003;61:1115-7.

7. Wellington K, Goa KL. Zoledronic acid: a review of its use in the management of bone metastases and hypercalcaemia of malignancy. Drugs 2003;63:417-37.

8. Ruggiero SL, Dodson TB, Fantasia J, Goodday R, Aghaloo T, Mehrotra B, et al. American Association of Oral and Maxillofacial Surgeons position paper on medication-related osteone-

crosis of the jaw: 2014 update. J Oral Maxillofac Surg 2014;72: 1938-56

9. Shibahara T. Antiresorptive agent-related osteonecrosis of the jaw (ARONJ): a twist of fate in the bone. Tohoku J Exp Med 2019;247:75-86.

10. Slough CM, Woo BM, Ueeck BA, Wax MK. Fibular free flaps in the management of osteomyelitis of the mandible. Head Neck 2008;30:1531-4.

11. Pincus DJ, Armstrong MB, Thaller SR. Osteomyelitis of the craniofacial skeleton. Semin Plast Surg 2009;23:73-9.

12. Shin J, Park SI, Cho JT, Jung SN, Byeon J, Seo BF. Necrotizing fasciitis of the masticator space with osteomyelitis of the mandible in an edentulous patient. Arch Craniofac Surg 2019;20: 270-3.

13. Hundepool AC, Dumans AG, Hofer SO, Fokkens NJ, Rayat SS, van der Meij EH, et al. Rehabilitation after mandibular reconstruction with fibula free-flap: clinical outcome and quality of life assessment. Int J Oral Maxillofac Surg 2008;37:1009-13.

14. Hidalgo DA. Fibula free flap: a new method of mandible reconstruction. Plast Reconstr Surg 1989;84:71-9.

15. Lee YW, You HJ, Jung JA, Kim DW. Mandibular reconstruction using customized three-dimensional titanium implant. Arch Craniofac Surg 2018;19:152-6.

16. Suh JM, Chung CH, Chang YJ. Head and neck reconstruction using free flaps: a 30-year medical record review. Arch Craniofac Surg 2021;22:38-44.

17. Chung JH, Kim KJ, Jung KY, Baek SK, Park SH, Yoon ES. Recipient vessel selection for head and neck reconstruction: a 30year experience in a single institution. Arch Craniofac Surg 2020;21:269-75.

18. Ren W, Gao L, Li S, Chen C, Li F, Wang Q, et al. Virtual planning and $3 \mathrm{D}$ printing modeling for mandibular reconstruction with fibula free flap. Med Oral Patol Oral Cir Bucal 2018;23: e359-66. 